\title{
Development of Fast Pixelated STEM Detector and its Applications using 4- Dimensional Dataset
}

Ryusuke Sagawa ${ }^{1}$, Hao Yang ${ }^{2}$, Lewys Jones ${ }^{3}$, Martin Simson ${ }^{4}$, Martin Huth ${ }^{4}$, Heike Soltau ${ }^{4}$, Peter D. Nellist $^{3}$ and Yukihito Kondo ${ }^{1}$

1. EM Business Unit, JEOL Ltd., Tokyo, Japan.

2. National Center for Electron Microscopy, Lawrence Berkeley National Laboratory, California, USA.

3. Department of Materials, University of Oxford, Oxford, UK.

4. PNDetector, PNDetector GmbH, München, Germany

In scanning transmission electron microscopy (STEM), one can obtain a variety of STEM images such as annular bright-field (ABF), annular dark-field (ADF) and differential phase contrast (DPC) images by changing the shapes of the scintillators of the detectors [1,2]. However, the intensity distribution in the convergent beam electron diffraction (CBED) pattern, which is projected on the plane of scintillator, is not fully utilized using the conventional detectors as they integrate the intensity over the scintillator. Meanwhile, direct electron detectors with fast frame rate and several ten thousand pixels have recently been commercialized and used in scanning electron microscopy [3-6]. Such detectors, when used for recording CBED patterns for each STEM probe position, are called pixelated STEM detectors. With the obtained 4-dimensional (4D) dataset, any type of STEM image can be synthesized in a post or real time processing using a user-defined selection of the integration area.

We have developed a pixelated STEM detector (JEOL, 4DCanvas ${ }^{\mathrm{TM}}$ ) to open the gate of its wide applications. Figure 1(a) shows an appearance of the 4DCanvas. It uses a fast direct electron CCD image sensor (PNDetector, pnCCD) which has a maximum readout speed of 4,000 fps and works at 30-300 kV. The detector is integrated into JEOL's aberration corrected commercial microscope (JEM-ARM200F), and users can record a 4D dataset by simply clicking a record button on microscope's software GUI. Live view of a CBED pattern and a synthesized STEM image are displayed during the acquisition of a STEM image. An example is shown in Fig. 1(b). Variety of STEM images (BF, ABF, DPC and annular DPC) can be easily obtained with the selections of integration areas on the CBED pattern. The detector is installed below the conventional ADF detector and has a retractable mechanism. Therefore, a simultaneous recording of a 4D dataset and an ADF-STEM image is enabled, and the other camera can be mounted at the opposite side. An EELS spectrometer can also be installed on the column.

One of the applications using a 4D dataset is an image processing technique called ptychography, which has been shown to provide high efficiency for reconstructing the phase image of an object $[7,8]$. Very recently, not only retrieving phase information, but also lens aberration correction work has been performed using ptychography technique [9].

Figure 2 shows a comparison between (a) an ADF STEM image and (b) a ptychographically reconstructed phase image of monolayer graphene. They are simultaneously recorded by the conventional ADF detector and the pixelated detector. Since the probe current was approximately as low as $1 \mathrm{pA}$, the ADF image is noisy and carbon atoms are barely visible. In contrast, the phase image shows high contrast with low noise and the carbon atomic positions can be unambiguously determined. It is clearly advantageous to use ptychography for this kind of sample (ideally thin) observation since the method effectively extracts signal components of the phase contrast of the sample. We believe that the 
developed pixelated detector 4DCanvas has a wide variety of potential other than previously reported applications and that the canvas for this detector has a lot of room to be painted with various colors.

\section{References:}

[1] E Okunishi et al., Microsc. Microanal., 15 (2009) p. 164.

[2] N Shibata et al., J. Electron Microsc., 59 (2010) p. 473.

[3] H Ryll et al., J. Instrum., 11 (2016) P04006.

[4] AR Lupini, M Chi and S Jesse, J. Microsc., 263 (2016) p. 43.

[5] MW Tate et al., Microsc. Microanal., 22 (2016) p. 237.

[6] M Krajnak et al., Ultramicroscopy, 165 (2016) p. 42.

[7] PD Nellist, B.C. McCallum and J.M. Rodenburg, Nature, 374 (1995) p. 630.

[8] TJ Pennycook et al., Ultramicroscopy, 151 (2015) p. 160.

[9] H Yang et al., Nature Communications, 7 (2016) p. 12532.
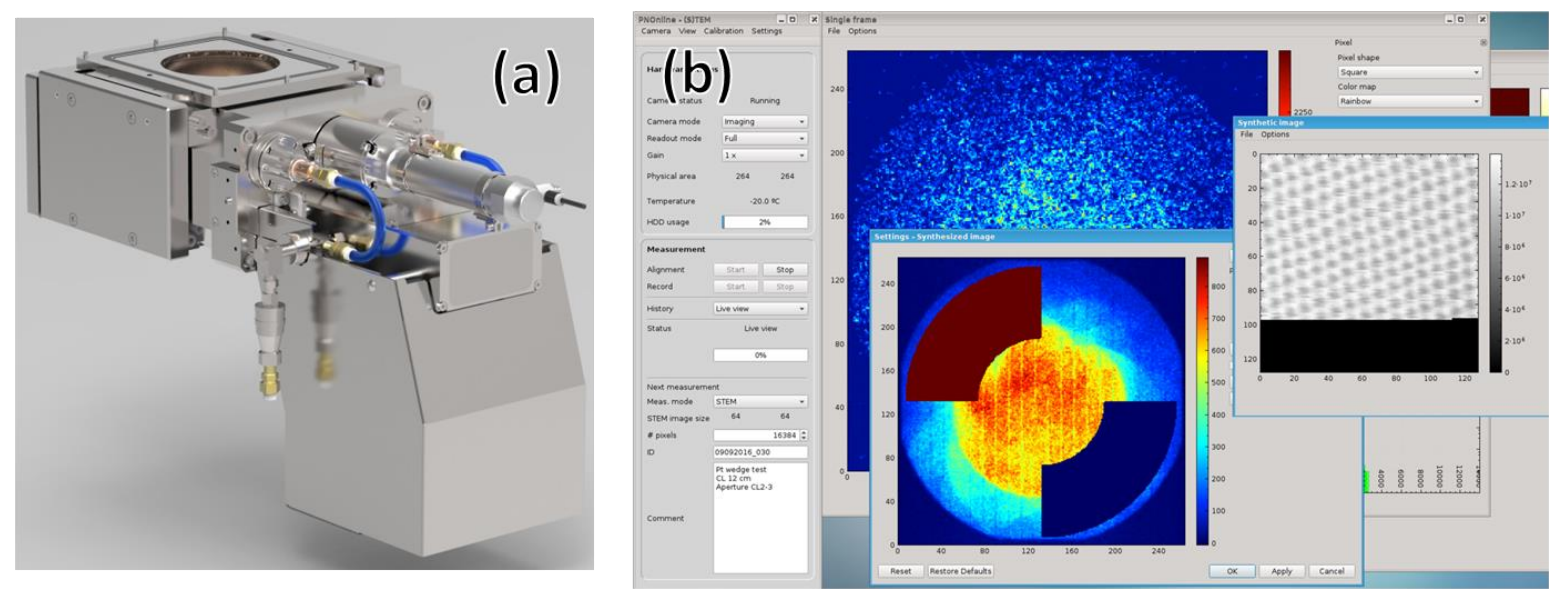

Figure 1. (a) Appearance of 4DCanvas camera. The CCD image sensor is retractable by air cylinders. (b) A screen shot of GUI on the control PC display. Users can control the camera settings such as gain and binning. Live view of CBED pattern (bottom center) and synthesized annular DPC STEM image (middle right) are displayed.
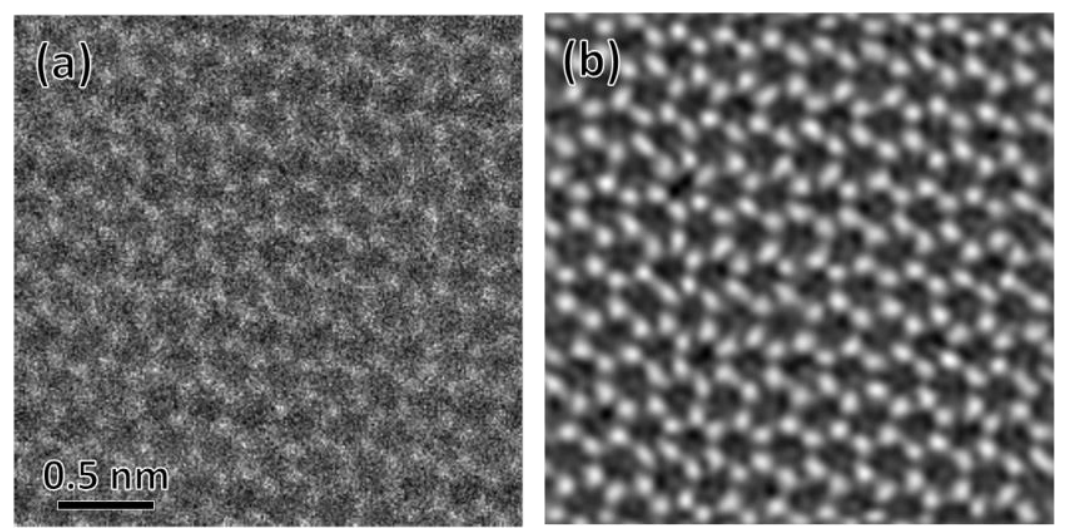

Figure 2. (a) and (b) show an ADF and a ptychographically reconstructed phase images of graphene, respectively, obtained simultaneously at $80 \mathrm{kV}$. The dwell time for a pixel and the total recording time for the images were $0.5 \mathrm{~ms}$ and $32.8 \mathrm{sec}$, respectively, which is realized with the fast readout of the 4DCanvas of 2,000 fps. 